\title{
Aggressive Quarantine Measures Reduce the High Morbidity of COVID-19 in Patients on Maintenance Hemodialysis and Medical Staff of Hemodialysis Facilities in Wuhan, China
}

\author{
Junhua $\mathrm{Li}^{\mathrm{a}}$ Yi Yang $^{\mathrm{a}}$ Meiling Gong ${ }^{\mathrm{a}}$ Jia Shi $^{\mathrm{a}} \mathrm{Xi}_{\text {Zhou }}{ }^{\mathrm{b}}$ Xue Xing $^{\mathrm{a}}$ \\ Hao Pan ${ }^{a}$ Shuiming Guo ${ }^{a}$ Xiaoyan Chang ${ }^{a}$ Anying Cheng ${ }^{a}$ Yanan Wang ${ }^{a}$

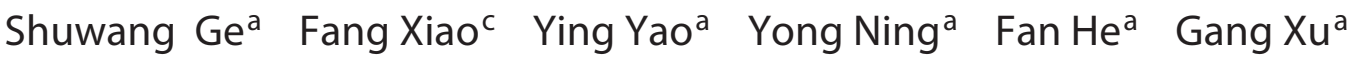 \\ a Department of Nephrology, Tongji Hospital Affiliated to Tongji Medical College, Huazhong University of Science \\ and Technology, Wuhan, China; ${ }^{b}$ State Key Laboratory of Virology, Wuhan Institute of Virology, Center for Biosafety \\ Mega-Science, Chinese Academy of Sciences, Wuhan, China; ' $D$ Department of Gastroenterology, Tongji Hospital \\ Affiliated to Tongji Medical College, Huazhong University of Science and Technology, Wuhan, China
}

\section{Keywords}

Maintenance hemodialysis · COVID-19 - SARS-CoV-2 .

Infection - Quarantine

\begin{abstract}
Introduction: Maintenance hemodialysis (MHD) patients are highly vulnerable to severe acute respiratory syndrome coronavirus 2 (SARS-CoV-2) infection. Medical staff of dialysis facilities without sufficient biosecurity protection are susceptible once exposed to asymptomatic coronavirus disease 2019 (COVID-19) patients. This study evaluated the epidemiological characteristics of COVID-19 in all MHD patients and medical staff of dialysis facilities in Wuhan, China. Methods: We collected COVID-19 morbidity and mortality data from MHD patients and medical staff from 52 hemodialysis centers in Wuhan. Then, we analyzed the symptoms and signs of patients and staff in our hospital (Tongji Hospital in Wuhan), and chest CT, SARS-CoV-2 nucleic acid detection and laboratory tests were performed. After aggressive quarantine of the COVID-19 patients, we followed up on the prog-
\end{abstract}

nosis of them. Results: We analyzed the hemodialysis data from Wuhan and found that $10 \%$ of MHD patients and $6.0 \%$ of medical staff were suspected of COVID-19. Further detection of SARS-CoV-2 nucleic acid showed that $1.7 \%$ of MHD patients and $2.9 \%$ of medical staff were confirmed as having COVID-19. In our facility, $18.9 \%(46 / 244)$ of patients and $9.5 \%$ $(6 / 63)$ of medical staff were suspected of COVID-19. Among them, $2.9 \%(7 / 244)$ of MHD patients and $4.8 \%$ (3/63) of medical staff tested positive for SARS-CoV-2 were confirmed as having COVID-19. Interestingly, $87.0 \%$ of MHD patients suspected of COVID-19 did not have obvious symptoms, but the CT screening showed features of viral pneumonia. There were no significant differences in symptoms, CT findings, comorbidity and laboratory findings of SARS-CoV-2 nucleic-acid-positive and -negative patients. We followed up these patients and found that 57 patients with COVID-19 died (COVID-19 mortality 8.9\%). Two patients from our dialysis center

Junhua Li, Yi Yang, and Meiling Gong contributed equally to this work.

$\begin{array}{ll}\text { karger@karger.com } & \text { C 2020 The Author(s) Karger } \\ \text { www.karger.com/kdd } & \text { Published by S. Karger AG, Basel Open caccess } \\ \text { This article is licensed under the Creative Commons Attribution- } & \text { NonCommercial-NoDerivatives 4.0 International License (CC BY- } \\ \text { NC-ND) (http://www.karger.com/Services/OpenAccessLicense). } \\ \text { Usage and distribution for commercial purposes as well as any dis- } \\ \text { tribution of modified material requires written permission. }\end{array}$

Fan He or Gang Xu

Department of Nephrology, Tongji Hospital Affiliated to Tongji Medical College Huazhong University of Science and Technology 1095 Jiefang Ave., Wuhan 430030 (China)

fhe@ tjh.tjmu.edu.cn or xugang@ tjh.tjmu.edu.cn 
with COVID-19 (mortality 4.3\%) died. No new infections occurred in our dialysis center after aggressive quarantine was initiated. Conclusions: The SARS-CoV-2 infection rates in MHD patients and medical staff in dialysis facilities were both high in Wuhan. Frequent chest CT and SARS-CoV-2 nucleic acid detection were needed to screen COVID-19 patients in dialysis facilities. Through the lessons of this experience on the aggressive diagnosis and quarantine measures of COVID-19 patients, we hope medical staff avoid more infections in serious epidemic areas.

(c) 2020 The Author(s) Published by S. Karger AG, Basel

Wuhan was one of the most seriously affected cities by the epidemic of coronavirus disease 2019 (COVID-19). In the early stage of the epidemic, many patients came to the Fever Clinic, and the medical staff lacked personal protection equipment, which caused a large number of medical staff members to be infected with COVID-19 in January 2020. However, the severe acute respiratory syndrome coronavirus 2 (SARS-CoV-2) nucleic acid detection kits were relatively insensitive, with low sensitivity in the early stage, which caused lots of missed diagnoses. Chest CT screening for the patients was much more liable to be performed in China (inexpensive and fast results). In the background of this abominable situation, the New Coronavirus Pneumonia Prevention and Control Program (5th edition, in Chinese), published by the National Health Commission of China [1], recommended that the clinical diagnosis of COVID-19 patients include a thorough history of epidemiology (residential location, close contact with COVID-19 patient), clinical symptoms, lymphocyte count and chest CT screening, regardless of whether SARSCoV-2 nucleic acid detection was positive or negative. After that, all patients clinically diagnosed with COVID-19 were quarantined. According to the latest diagnostic guidelines issued by the National Health Commission of China (7th edition) [2], these patients with clinical diagnosis of COVID-19 (suspected cases) need to be further tested for SARS-CoV-2 nucleic acid, antiSARS-CoV-2 antibody and blood routine etc. The patients with positive nucleic acid were finally confirmed as COVID-19 and transferred to the designated hospitals for further treatment. From these aggressive diagnostic criteria and quarantine measures in the early stage (from February), the epidemic of COVID-19 was controlled throughout the city of Wuhan, including among hemodialysis (HD) patients and medical staff in dialysis facilities.
End-stage renal disease is an increasingly burdensome disease worldwide, and approximately $63-89.1 \%$ of affected patients require maintenance hemodialysis (MHD) [3]. MHD patients in Wuhan were a special group because, during this epidemic, they could not be isolated at home as the general population. MHD patients and their accompanying family members had to travel back and forth between home and the hospital two to three times a week for HD treatment and stay for more than $4 \mathrm{~h}$ in closed and crowded HD centers. Therefore, highly susceptible MHD patients with a missed diagnosis of COVID-19 might become potential sources of infection. The clinical characteristics and epidemiology of SARS-CoV-2 infection in MHD patients in Wuhan are unclear.

Urgent questions need to be addressed to promptly discriminate potential infection and to quarantine to prevent HD patients and medical staff from infection. In this study, we aimed to describe the epidemiological, clinical, laboratory, and radiological characteristics of COVID-19 in MHD patients and medical staff. We hope to attract attention to this specific population and reduce the infection rate through aggressive diagnosis and quarantine of $\mathrm{CO}$ VID-19 patients and medical staff during this epidemic.

\section{Methods}

Study Design, Setting and Participation

From February 1 to February 14, 2020, we collected data from 52 dialysis facilities in Wuhan from the Hemodialysis Quality Control Center of Wuhan, including the name of the hospitals and the numbers of MHD patients and medical staff with chest CT screening and SARS-CoV-2 nucleic acid detection, and we followed up on the survival outcome of these individuals.

All MHD patients and medical staff in three HD centers were screened, including Tongji Hospital Main Campus, Optical Valley Branch, and Sino-French New City Branch of Tongji Hospital Affiliated with Tongji Medical College, Huazhong University of Science and Technology. We collected data on the history of exposure, symptoms and signs for all subjects. In addition, routine blood tests, blood biochemistry analysis, hypersensitive CRP (hCRP) analysis, chest CT screening and SARS-CoV-2 nucleic acid detection (Chinese Center for Disease Control and Prevention [CDC] recommended kit [BioGerm, Shanghai, China]) of nasopharyngeal swab specimens were performed for all dialysis patients $[4,5]$.

All information was obtained and managed through established data collection forms. We reviewed clinical records, laboratory findings and chest CT scans for all enrolled HD patients. All data were checked by two physicians (Junhua Li and Hao Pan). All patients were diagnosed by consultation through three COVID-19 experts from our hospital according to the New Coronavirus Pneumonia Prevention and Control Program (7th edition, in Chinese), published by the National Health Commission of China. 


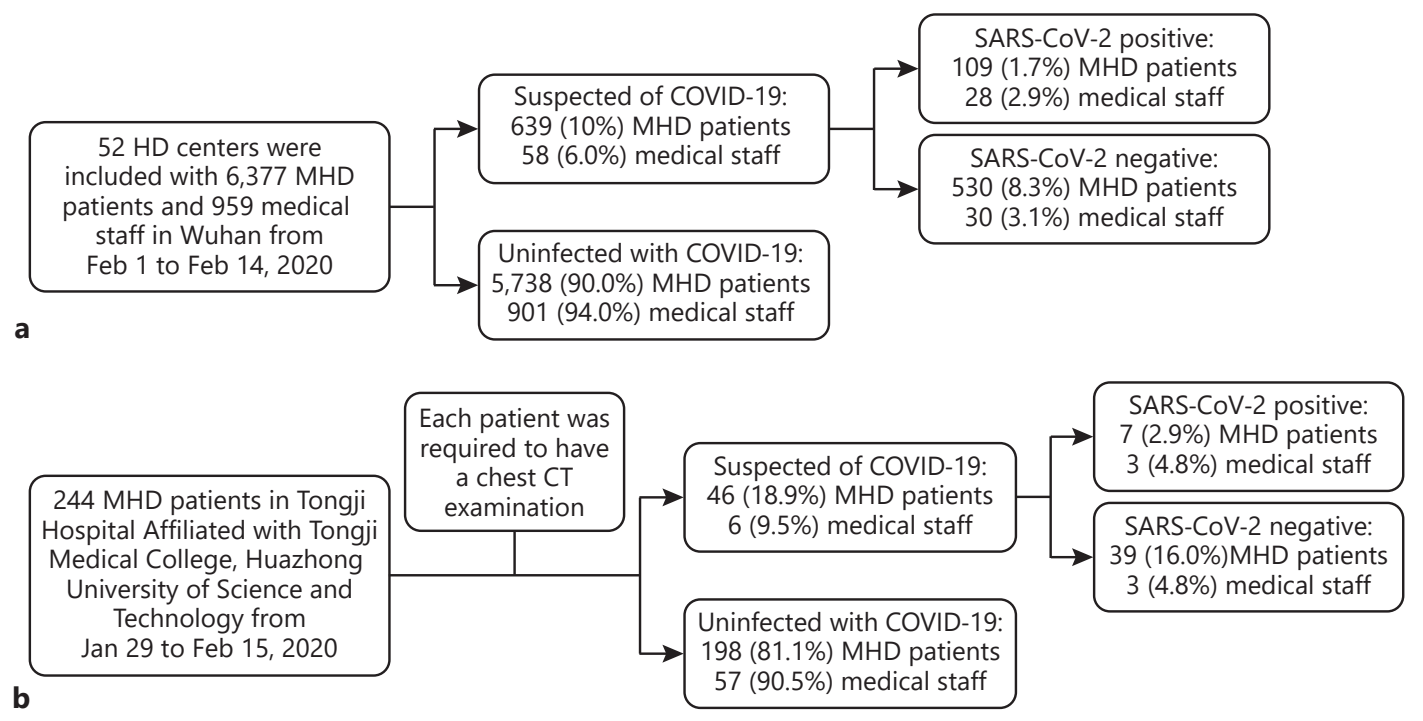

Fig. 1. Flowchart showing recruitment of participants and grouping. a Recruitment of participants in 52 HD centers in Wuhan. b Recruitment of participants and grouping in our hospital.

\section{Procedures and Management}

The demographic and epidemiological data, signs and symptoms, laboratory results and radiological characteristics were reviewed. The results of patients suspected of COVID-19 were compared with those of uninfected patients from our hospital. Subsequently, all patients with suspected infection were tested for SARS-CoV-2 nucleic acid, and the data of nucleic-acid-positive and -negative patients were compared.

In dialysis facilities, all dialysis patients suspected of COVID-19 were isolated in a dedicated area of the last shift of the dialysis units to separate them from uninfected patients, and patients with positive SARS-CoV-2 nucleic acid were transferred to COVID-19-designated hospitals. The medical staff treated them with standard protection during dialysis treatment. We treated these suspected patients with Lianhua Qingwen capsules and Jinye Baidu granule (traditional Chinese medicine). Beyond that, when the patients became worse or critical, they were sent to isolation wards in COVID-19-designated hospitals.

\section{Outcomes}

We followed up the cases of suspected COVID-19 patients with prognosis in Wuhan dialysis facilities until April 1, 2020. The survival of the patients was analyzed, and chest CT and laboratory tests for routine blood parameters were also performed repeatedly in our hospital.

\section{Definition}

In the data of our hospital, patients with clinical diagnosis of COVID-19 (suspected cases) refer to the typical manifestations of viral pneumonia on the chest CT scan of the patient without SARSCoV-2 nucleic acid detection; patients confirmed as having COVID-19 refer to the typical manifestations of viral pneumonia on the chest CT scan and were tested SARS-CoV-2 nucleic acid positive.
Statistical Analyses

GraphPad Prism 6 (GraphPad Software, USA) and SPSS 23.0 (IBM Corporation, Armonk, NY, USA) were used for statistical analysis and visualization. To show the normal distribution of variables, the Kolmogorov-Smirnov test was used. Continuous parametric data were expressed as the mean (standard deviation), and the $t$ test was used for univariate comparisons. Continuous nonparametric data were expressed as the median (interquartile range), and the Kruskal-Wallis test was used for univariate comparisons. Categorical data were expressed as frequencies (percentages), and the $\chi^{2}$ test, Fisher's exact test and $\chi^{2}$ with Yates' correction were used to compare variables. A two-tailed $p<0.05$ was considered statistically significant.

\section{Results}

\section{HD Patients and Medical Staff in HD Facilities in Wuhan}

From February 1 to February 14, 2020, 52 HD centers from Wuhan were identified and included in this study. As shown in Figure 2a and b, a total of 6,377 MHD patients were eligible. There were 639 patients (10.0\%) suspected of COVID-19 in 52 dialysis facilities according to the data of the Hemodialysis Quality Control Center of Wuhan. Among them, 109 (1.7\%) HD patients were confirmed as having COVID-19 from 23 HD centers (44.2\%). All these patients suspected of COVID-19 were isolated in a dedicated area of the last shift of the dialysis unit, and 


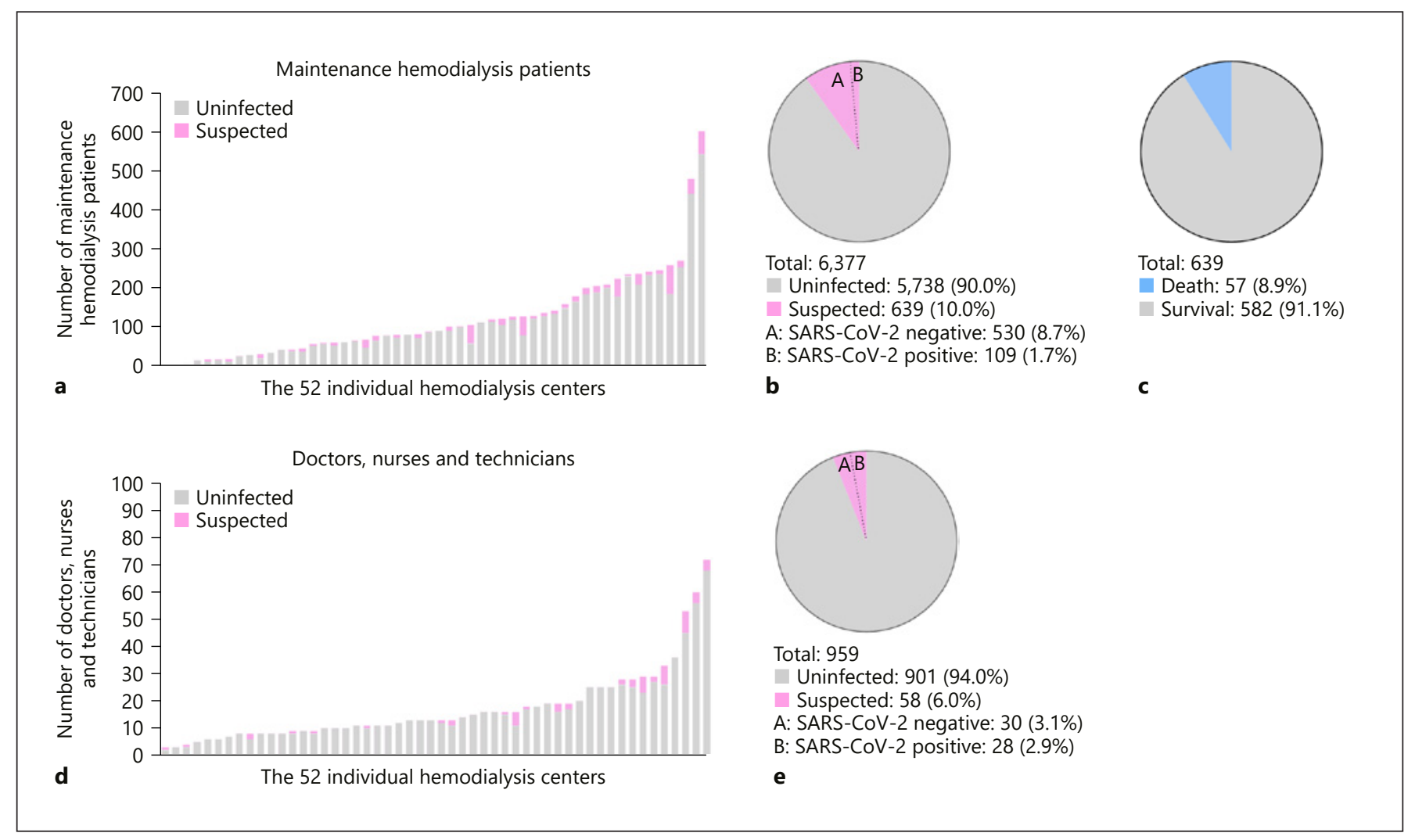

Fig. 2. Summary of COVID-19 epidemiological features in maintenance hemodialysis (MHD) patients and medical staff in 52 hemodialysis centers in Wuhan, China. a COVID-19 data of MHD patients in 52 dialysis centers. b SARS-CoV-2 infected and uninfected proportions in MHD patients. c Survival and mortality of MHD patients with COVID-19 in 52 dialysis centers. d COVID-19 data of medical staff in 52 dialysis centers. e SARS-CoV-2 infected and uninfected proportions in medical staff.

then after SARS-CoV-2 nucleic acid detection, patients confirmed as having COVID-19 and critically ill patients were transferred to COVID-19-designated hospitals (Fig. 1a, 2).

There were 959 medical staff (including doctors, nurses, and technicians) working in these 52 dialysis centers (Fig. 2d, e), and 58 (6\%) medical staff were suspected of COVID-19 and were quarantined and treated. Overall 28 (2.9\%) medical staffs were confirmed as having COVID-19 from 13 HD centers (25.0\%) and were transferred to COVID-19-designated hospitals (Fig. 1a, 2).

We followed up these patients and staff until April 1, 2020 , and there were 216 deaths (total mortality $3.4 \%$ ) of HD patients in Wuhan, including 57 due to COVID-19 (COVID-19 mortality 8.9\%) during this period (Fig. 2c). Among the medical staff analyzed, there were no deaths during this epidemic.
HD Patients and Medical Staff in Our Dialysis Center

By February 15, 2020, our study had enrolled a total of 244 MHD patients in our hospital (Fig. 1b). The median age of the patients was 55 years (range, 13-86), and the study population included 160 males $(65.6 \%)$ and $84 \mathrm{fe}-$ males (34.4\%). Among these patients, the primary disease causing renal failure was chronic nephritis [176 (72.1\%)], followed by diabetes mellitus [32 (13.1\%)] and hypertension [14 (5.7\%)]. Hypertension [216 (88.5\%)], diabetes [43 (17.6\%)], coronary atherosclerotic heart disease [16 $(6.6 \%)]$, and gout [9 (3.7\%)] were the most common coexisting diseases. HD/hemodiafiltration (HDF) [138 (56.8\%)], HD [72 (29.6\%)], and HDF [25 (10.3\%)] were the three most common dialysis modes (Table 1 ).

Of the $244 \mathrm{HD}$ patients, $46 \mathrm{HD}$ patients (18.9\%) were suspected of COVID-19 by expert consultation in our hospital, and the other 198 patients $(81.1 \%)$ were assigned to the COVID-19 uninfected group. There was no sig- 
Table 1. Demographics and clinical characteristics of maintenance hemodialysis patients between suspected of COVID-19 and uninfected with COVID-19

\begin{tabular}{|c|c|c|c|c|}
\hline Parameters & $\begin{array}{l}\text { Total } \\
(n=244)\end{array}$ & $\begin{array}{l}\text { Suspected of } \\
\text { COVID-19 } \\
(n=46)\end{array}$ & $\begin{array}{l}\text { Uninfected with } \\
\text { COVID-19 } \\
(n=198)\end{array}$ & $p$ \\
\hline Age & & & & $0.627^{\mathrm{b}}$ \\
\hline Mean, years & 55.0 & 60.0 & 55.0 & \\
\hline Standard deviation & $43.3-67.0$ & $44.8-66.0$ & $43.0-68.0$ & \\
\hline Sex & & & & $0.773^{\mathrm{a}}$ \\
\hline Male, $n(\%)$ & $160(65.6)$ & $31(67.4)$ & $129(65.2)$ & \\
\hline Female, $n(\%)$ & $84(34.4)$ & $15(32.6)$ & $69(34.8)$ & \\
\hline \multicolumn{5}{|l|}{ Symptoms } \\
\hline Fever, $n(\%)$ & $7(2.9)$ & $3(6.5)$ & $4(2.0)$ & $0.247^{\mathrm{d}}$ \\
\hline \multicolumn{5}{|l|}{ Primary disease } \\
\hline Chronic nephritis, $n(\%)$ & $176(72.1)$ & $28(60.9)$ & $148(74.7)$ & $0.059^{\mathrm{a}}$ \\
\hline Hypertension, $n(\%)$ & $14(5.7)$ & $2(4.3)$ & $12(6.1)$ & $0.922^{\mathrm{d}}$ \\
\hline Diabetes mellitus, $n(\%)$ & $32(13.1)$ & $10(21.7)$ & $22(11.1)$ & $0.054^{\mathrm{a}}$ \\
\hline Obstructive nephropathy, $n(\%)$ & $2(0.8)$ & $2(4.3)$ & 0 & $0.035^{* \mathrm{e}}$ \\
\hline Polycystic kidney, $n(\%)$ & $6(2.5)$ & $0(0)$ & $6(3.0)$ & $0.598^{\mathrm{e}}$ \\
\hline Gouty nephropathy, $n$ (\%) & $6(2.5)$ & $2(4.3)$ & $4(2.0)$ & $0.697^{\mathrm{d}}$ \\
\hline Lupus nephritis, $n(\%)$ & $7(2.9)$ & $2(4.3)$ & $5(2.5)$ & $0.860^{\mathrm{d}}$ \\
\hline Other, $n(\%)$ & $1(0.4)$ & 0 & $1(0.5)$ & $>0.9999^{\mathrm{e}}$ \\
\hline \multicolumn{5}{|l|}{ Any comorbidity } \\
\hline \multicolumn{5}{|l|}{ Heart disease, $n(\%)$} \\
\hline Tumor, $n(\%)$ & $1(0.4)$ & 0 & $1(0.5)$ & $>0.9999^{\mathrm{e}}$ \\
\hline Chronic hepatitis B virus, $n(\%)$ & $10(4.1)$ & $4(8.7)$ & $6(3.0)$ & $0.183^{\mathrm{d}}$ \\
\hline Chronic hepatitis $\mathrm{C}$ virus, $n(\%)$ & $2(0.8)$ & 0 & $2(1.0)$ & $>0.9999^{e}$ \\
\hline Syphilis, $n(\%)$ & $3(1.2)$ & $2(4.8)$ & $1(0.5)$ & $0.092^{\mathrm{e}}$ \\
\hline HIV infection, $n(\%)$ & 0 & 0 & 0 & - \\
\hline Gallbladder stone disease, $n(\%)$ & $14(5.7)$ & $5(10.9)$ & $9(4.5)$ & $0.190^{\mathrm{d}}$ \\
\hline Mode of dialysis & & & & $<0.0001^{\mathrm{a}}$ \\
\hline $\mathrm{HD}$ & $72(29.6)$ & $7(15.2)$ & $65(33.0)$ & \\
\hline $\mathrm{HDF}$ & $25(10.3)$ & $3(6.5)$ & $22(11.2)$ & \\
\hline $\mathrm{HD}+\mathrm{HDF}$ & $138(56.8)$ & $29(63.0)$ & $109(55.3)$ & \\
\hline $\mathrm{HD}+\mathrm{HP}$ & $1(0.4)$ & $1(2.2 \%)$ & 0 & \\
\hline $\mathrm{HD}+\mathrm{HDF}+\mathrm{HP}$ & $7(2.9)$ & $6(13.0)$ & $1(0.5)$ & \\
\hline
\end{tabular}


Table 1 (continued)

\begin{tabular}{|c|c|c|c|c|}
\hline Parameters & $\begin{array}{l}\text { Total } \\
(n=244)\end{array}$ & $\begin{array}{l}\text { Suspected of } \\
\text { COVID-19 } \\
(n=46)\end{array}$ & $\begin{array}{l}\text { Uninfected with } \\
\text { COVID-19 } \\
(n=198)\end{array}$ & $p$ \\
\hline \multicolumn{5}{|l|}{ Laboratory findings } \\
\hline White blood cell count, median (IQR), $10^{9} / \mathrm{L}$ & $5.7(4.7-6.7)$ & $6.3(5.2-7.1)$ & $5.5(4.7-6.7)$ & $0.121^{\mathrm{b}}$ \\
\hline Neutrophil ratio, median (IQR), \% & $69.6(64.1-74.3)$ & $73.0(68.1-78.3)$ & $68.7(63.5-73.7)$ & $0.017^{*, \mathrm{~b}}$ \\
\hline Neutrophil count, median (IQR), $10^{9} / \mathrm{L}$ & $4.0(3.2-4.7)$ & $4.5(3.7-5.0)$ & $3.8(3.1-4.6)$ & $0.038^{*, \mathrm{~b}}$ \\
\hline Lymphocyte ratio, mean (SD), \% & $19.2(7.0)$ & $14.9(5.5)$ & $20.1(6.9)$ & $0.001^{* *, c}$ \\
\hline Lymphocyte count, median (IQR), $10^{9} / \mathrm{L}$ & $1.0(0.8-1.3)$ & $0.9(0.6-1.2)$ & $1.0(0.8-1.3)$ & $0.033^{*, \mathrm{~b}}$ \\
\hline Monocyte ratio, median (IQR), \% & $7.5(6.3-9.1)$ & $7.7(6.7-9.3)$ & $7.4(6.1-8.9)$ & $0.330^{\mathrm{b}}$ \\
\hline Monocyte count, median (IQR), $10^{9} / \mathrm{L}$ & $0.4(0.3-0.6)$ & $0.5(0.4-0.6)$ & $0.4(0.3-0.6)$ & $0.113^{\mathrm{b}}$ \\
\hline Hemoglobin, mean (SD), g/L & $114.8(18.0)$ & $112.7(20.4)$ & $115.3(17.5)$ & $0.538^{\mathrm{c}}$ \\
\hline Platelet, mean (SD), $10^{9} / \mathrm{L}$ & $173.8(58.6)$ & $172.5(52.6)$ & $174.1(60.0)$ & $0.904^{\mathrm{c}}$ \\
\hline Alanine aminotransferase, median (IQR), U/L & $8.0(5.0-12.0)$ & $12.0(6.0-18.0)$ & $8.0(5.0-11.0)$ & $0.022^{*, \mathrm{~b}}$ \\
\hline Aspartate aminotransferase, median (IQR), U/L & $12.0(8.0-17.0)$ & $14.0(8.0-17.0)$ & $11.5(8.3-16.8)$ & $0.695^{\mathrm{b}}$ \\
\hline Total protein, mean (SD), g/L & $67.4(4.8)$ & $67.0(5.3)$ & $67.5(4.7)$ & $0.720^{\mathrm{c}}$ \\
\hline Albumin, mean (SD), g/L & $39.2(4.3)$ & $38.6(5.5)$ & $39.4(4.0)$ & $0.545^{\mathrm{c}}$ \\
\hline Globulin, mean (SD), g/L & $28.2(4.3)$ & $28.3(3.8)$ & $28.2(4.4)$ & $0.895^{\mathrm{c}}$ \\
\hline Lactate dehydrogenase, median (IQR), U/L & $196.5(174.5-240.5)$ & $227.0(208.8-249.3)$ & $187.5(170.0-235.3)$ & $0.012^{*, \mathrm{~b}}$ \\
\hline Alkaline phosphatase, median (IQR), U/L & $80.0(66.5-96.0)$ & $83.0(61.3-103.8)$ & $80.0(68.0-95.0)$ & $0.921^{\mathrm{b}}$ \\
\hline Potassium, mean $(\mathrm{SD}), \mathrm{mmol} / \mathrm{L}$ & $5.3(0.8)$ & $5.2(0.7)$ & $5.3(0.8)$ & $0.562^{\mathrm{c}}$ \\
\hline Phosphorus, mean (SD), mmol/L & $2.0(0.8)$ & $1.9(0.6)$ & $2.0(0.8)$ & $0.437^{\mathrm{c}}$ \\
\hline hCRP, median (IQR), mg/L & $1.8(0.7-4.4)$ & $2.4(1.4-10.4)$ & $1.6(0.7-4.1)$ & $0.151^{\mathrm{b}}$ \\
\hline Parathyroid hormone, median (IQR), pg/mL & $306.5(188.2-552.7)$ & $292.0(234.3-573.6)$ & $315.3(187.0-552.7)$ & $0.889^{\mathrm{b}}$ \\
\hline
\end{tabular}

Values are expressed as mean (standard deviation), median (25th-75th percentile) or $n$ (\%). COVID-19, coronavirus disease 2019; $\mathrm{CT}$, computed tomography; IQR, interquartile range; SD, standard deviation; HD, hemodialysis, HDF, hemodiafiltration, HP, hemoperfusion; HIV, human immunodeficiency virus; hCRP, hypersensitive C-reactive protein. ${ }^{\mathrm{a}} \chi^{2}$ test. ${ }^{\mathrm{b}}$ Mann-Whitney $\mathrm{U}$ test. ${ }^{\mathrm{c}} t$ test. ${ }^{\mathrm{d}} \chi^{2}$ with Yates' correction. ${ }^{\text {e }}$ Fisher's exact test.

nificant difference in the median age between the patients suspected of COVID-19 and the uninfected patients (55.0 vs. $60.0, p>0.05)$ or in sex. Comparison symptoms between the patients suspected of COVID-19 and the uninfected patients showed the proportions of patients with fever [3 (6.5\%) vs. 4 (2.0), $p>0.05$ ], fatigue [ 4 (8.7\%) vs. $0, p<0.05]$, cough [2 (4.3\%) vs. $0, p<0.05]$, and expectoration $[2(4.3 \%)$ vs. $0, p<0.05]$. However, the symptoms were all rare, showing a proportion with no obvious symptoms up to 87 and $98 \%$ in the suspected group and uninfected group, respectively (Table 1).

Among the primary diseases, there was no difference between the two groups, but it was demonstrated that the proportion of patients having diabetes mellitus in the suspected COVID-19 group seemed higher than that in the uninfected group [10 (21.7\%) vs. $22(11.1 \%), p=0.054$ ] Among the comorbidities, the proportion of patients having hypertension was significantly different between the suspected COVID-19 group and the uninfected group [28 (60.9\%) vs. $188(94.9 \%), p<0.05]$, but there was no difference in other comorbidities, including diabetes mellitus. In the analysis of dialysis modes, the COVID-19 group was different from the uninfected group $(p<0.05)$, but the data were limited; therefore, we could not determine which mode was better to prevent infection. The proportion of patients undergoing HDF in the uninfected group (22 [11.2\%]) seemed much higher than that in the suspected COVID-19 group (3 [6.5\%]) (Table 1).

Regarding laboratory tests, there were significant differences between the suspected COVID-19 group and the uninfected group in neutrophil ratio (73.0 vs. $68.7 \%, p<$ $0.05)$, neutrophil count $\left(4.5 \times 10^{9} / \mathrm{L}\right.$ vs. $3.8 \times 10^{9} / \mathrm{L}, p<$ 0.05 ), lymphocyte ratio ( 14.9 vs. $20.1 \%, p<0.05$ ), lymphocyte count $\left(0.90 \times 10^{9} / \mathrm{L}\right.$ vs. $\left.1.0 \times 10^{9} / \mathrm{L}, p<0.05\right)$ and lactate dehydrogenase level (227.0 vs. $187.5 \mathrm{U} / \mathrm{L}, p<0.05)$. However, no difference was found between the COVID-19 group and the uninfected group in white blood cell count $\left(6.3 \times 10^{9} / \mathrm{L}\right.$ vs. $\left.5.5 \times 10^{9} / \mathrm{L}, p>0.05\right)$, hemoglobin level (112.7 vs. $115.3 \mathrm{~g} / \mathrm{L}, p>0.05)$, platelet counts $(172.5$ $\times 10^{9} / \mathrm{L}$ vs. $174.1 \times 10^{9} / \mathrm{L}, p>0.05$ ), albumin level (38.6 vs. 
Table 2. Comparison of demographics and clinical characteristics of SARS-CoV-2 positive group and SARS-CoV-2 negative group in suspected COVID-19 maintenance hemodialysis patients

\begin{tabular}{|c|c|c|c|c|}
\hline Parameters & $\begin{array}{l}\text { All patients } \\
(n=46)\end{array}$ & $\begin{array}{l}\text { SARS-CoV-2 } \\
\text { positive }(n=7)\end{array}$ & $\begin{array}{l}\text { SARS-CoV-2 } \\
\text { negative }(n=39)\end{array}$ & $p$ \\
\hline Age & & & & $0.994^{\mathrm{b}}$ \\
\hline IQR, years & $44.8-66.0$ & $48.0-66.0$ & $44.0-70.0$ & \\
\hline Range, years & $23-81$ & $39-66$ & $39-66$ & \\
\hline Female, $n(\%)$ & $15(32.6)$ & $3(42.9)$ & $12(30.8)$ & \\
\hline \multicolumn{5}{|l|}{ Symptoms } \\
\hline Fever, $n(\%)$ & $3(6.5)$ & $1(14.3)$ & $2(5.1)$ & $0.398^{\mathrm{e}}$ \\
\hline Fatigue, $n(\%)$ & $4(8.7)$ & 0 & $4(10.3)$ & $>0.9999^{\mathrm{e}}$ \\
\hline Cough, $n(\%)$ & $2(4.3)$ & 0 & $2(5.1)$ & $>0.9999^{\mathrm{e}}$ \\
\hline \multicolumn{5}{|l|}{ CT findings } \\
\hline Unilateral lung, $n(\%)$ & $16(34.8)$ & $3(42.9)$ & $13(33.3)$ & $0.955^{\mathrm{d}}$ \\
\hline Bilateral lung, $\mathrm{n}(\%)$ & $30(65.2)$ & $4(57.1)$ & $26(66.7)$ & $0.955^{\mathrm{d}}$ \\
\hline \multicolumn{5}{|l|}{ Lobes } \\
\hline One lobes, $n(\%)$ & $13(28.3)$ & $2(28.6)$ & $11(28.2)$ & $0.663^{\mathrm{d}}$ \\
\hline Two lobes, $n(\%)$ & $8(17.4)$ & 0 & $8(20.5)$ & $0.325^{\mathrm{e}}$ \\
\hline Three lobes, $n(\%)$ & $6(13.0)$ & $1(14.3)$ & $5(12.8)$ & $>0.9999^{\mathrm{e}}$ \\
\hline Four lobes, $n(\%)$ & $2(4.8)$ & 0 & $2(5.1)$ & $>0.9999^{\mathrm{e}}$ \\
\hline Five lobes, $n(\%)$ & $17(37.0)$ & $4(57.1)$ & $13(33.3)$ & $0.438^{\mathrm{d}}$ \\
\hline \multicolumn{5}{|l|}{ Involved lung zones } \\
\hline Upper lobes, $n(\%)$ & $37(80.4)$ & $5(71.4)$ & $32(82.1)$ & $0.893^{\mathrm{d}}$ \\
\hline Pleural thickening, $n(\%)$ & $14(30.4)$ & $4(57.1)$ & $10(25.6)$ & $0.222^{\mathrm{d}}$ \\
\hline Pleural effusion, $n(\%)$ & $14(30.4)$ & $3(42.9)$ & $11(28.2)$ & $0.742^{\mathrm{d}}$ \\
\hline Unilateral, $n(\%)$ & $7(15.2)$ & $1(14.3)$ & $6(15.4)$ & $0.619^{\mathrm{d}}$ \\
\hline Bilateral, $n(\%)$ & $7(15.2)$ & $2(28.6)$ & $5(12.8)$ & $0.619^{\mathrm{d}}$ \\
\hline Aortic calcification, $n(\%)$ & $26(56.5)$ & $2(28.6)$ & $24(61.3)$ & $0.228^{\mathrm{d}}$ \\
\hline Coronary artery calcification, $n(\%)$ & $26(56.5)$ & $3(42.9)$ & $23(59.0)$ & $0.705^{\mathrm{d}}$ \\
\hline Pericardial effusion, $n(\%)$ & $12(26.1)$ & $4(57.1)$ & $8(20.5)$ & $0.118^{\mathrm{d}}$ \\
\hline Left atrial enlargement, $n(\%)$ & $12(26.1)$ & $3(42.9)$ & $9(23.1)$ & $0.529^{\mathrm{d}}$ \\
\hline Left ventricular enlargement, $n(\%)$ & $11(23.9)$ & $3(42.9)$ & $8(20.5)$ & $0.427^{\mathrm{d}}$ \\
\hline
\end{tabular}


Table 2 (continued)

\begin{tabular}{|c|c|c|c|c|}
\hline Parameters & $\begin{array}{l}\text { All patients } \\
(n=46)\end{array}$ & $\begin{array}{l}\text { SARS-CoV-2 } \\
\text { positive }(n=7)\end{array}$ & $\begin{array}{l}\text { SARS-CoV-2 } \\
\text { negative }(n=39)\end{array}$ & $p$ \\
\hline \multicolumn{5}{|l|}{ Any comorbidity } \\
\hline Diabetes, $n(\%)$ & $11(23.9)$ & $3(42.9)$ & $8(20.5)$ & $0.427^{\mathrm{d}}$ \\
\hline Heart disease, $n(\%)$ & $4(8.7)$ & 0 & $4(10.3)$ & $>0.9999^{\mathrm{e}}$ \\
\hline Malignancy, $n(\%)$ & 0 & 0 & 0 & - \\
\hline Chronic hepatitis $\mathrm{C}$ virus, $n(\%)$ & 0 & 0 & 0 & - \\
\hline Syphilis, $n(\%)$ & $2(4.8)$ & 0 & $2(5.1)$ & $>0.9999^{\mathrm{e}}$ \\
\hline HIV infection, $n(\%)$ & 0 & 0 & 0 & - \\
\hline Gallbladder stone disease, $n(\%)$ & $5(10.9)$ & 0 & $5(12.8)$ & $>0.9999^{\mathrm{e}}$ \\
\hline \multicolumn{5}{|l|}{ Dialysis } \\
\hline Dialysis route & & & & $>0.9999^{d}$ \\
\hline $\mathrm{HDF}, n(\%)$ & $3(6.5)$ & 0 & $3(7.7)$ & \\
\hline $\mathrm{HD}+\mathrm{HDF}, n(\%)$ & $29(63.0)$ & $4(57.1)$ & $25(64.1)$ & \\
\hline $\mathrm{HD}+\mathrm{HP}, n(\%)$ & $1(2.2 \%)$ & 0 & $1(2.6)$ & \\
\hline $\mathrm{HD}+\mathrm{HDF}+\mathrm{HP}, n(\%)$ & $6(13.0)$ & $2(28.6)$ & $4(10.3)$ & \\
\hline Anticoagulant used in hemodialysis & & & & $>0.9999^{\mathrm{e}}$ \\
\hline Heparin, $n(\%)$ & $40(95.2)$ & $7(100)$ & $33(84.6)$ & \\
\hline No heparin, $n(\%)$ & $2(4.8)$ & 0 & $2(5.7)$ & \\
\hline Dehydration, mean (SD), L & $2.2(1.0)$ & $1.8(0.8)$ & $2.3(1.1)$ & $0.250^{\mathrm{c}}$ \\
\hline \multicolumn{5}{|l|}{ Laboratory findings } \\
\hline White blood cell count, median (IQR), $10^{9} / \mathrm{L}$ & $6.3(5.2-7.1)$ & $5.4(2.6-6.4)$ & $6.5(5.5-7.9)$ & $0.151^{\mathrm{b}}$ \\
\hline Neutrophil ratio, median (IQR), \% & $73.0(68.1-78.3)$ & $77.0(64.5-87.8)$ & $72.8(68.1-75.3)$ & $0.442^{\mathrm{b}}$ \\
\hline Potassium, mean (SD), mmol/L & $5.2(0.7)$ & $5.1(0.5)$ & $5.2(0.8)$ & $0.938^{c}$ \\
\hline Phosphorus, mean (SD), mmol/L & $1.9(0.6)$ & $1.6(0.4)$ & $2.0(0.6)$ & $0.257^{\mathrm{c}}$ \\
\hline
\end{tabular}

Values are expressed as mean (SD), median (25th-75th percentile) or $n$ (\%). COVID-19, coronavirus disease 2019; CT, computed tomography; IQR, interquartile range; SD, standard deviation; HD, hemodialysis, HDF, hemodiafiltration, HP, hemoperfusion. ${ }^{\mathrm{a}} \chi^{2}$ test. ${ }^{\mathrm{b}}$ Mann-Whitney U test. ${ }^{\mathrm{c}} t$ test. ${ }^{\mathrm{d}} \chi^{2}$ with Yates' correction. ${ }^{\mathrm{e}}$ Fisher's exact test.

$39.4 \mathrm{~g} / \mathrm{L}, p>0.05)$, or hCRP level (2.4 vs. $1.6 \mathrm{mg} / \mathrm{L}, p>$ 0.05) (Table 1).

These patients suspected of COVID-19 were tested for SARS-CoV-2 nucleic acid. 15.2\% (7/46) tested positive for SARS-CoV-2 nucleic acid, accounting for $2.9 \%$ of the total study population. In 7 SARS-CoV-2 nucleic-acidpositive patients, only 1 had fever, and the remaining 6 had no obvious symptoms. Interestingly, more than $80 \%$ of MHD patients in the SARS-CoV-2 nucleic-acid-posi- tive or -negative group did not have any obvious clinical symptoms (Table 2).

The CT screenings all showed features of viral pneumonia in the patients suspected of COVID-19. Sixteen (34.8\%) MHD patients suspected of COVID-19 showed unilateral pneumonia on chest CT, and the remaining $30(65.2 \%)$ patients showed bilateral pneumonia. The affected lung lobes ranged from 1 to 5, with 17 (37\%) patients with all 5 lobes infected. For the involved lung zones, the infection rates of 
Table 3. Comparison of MHD patients and medical staff suspected of COVID-19 before and after the quarantine period

\begin{tabular}{|c|c|c|}
\hline Parameters & Before quarantine & After quarantine \\
\hline MHD patients, $\mathrm{n}$ & $n=46$ & $n=44$ \\
\hline \multicolumn{3}{|l|}{ Clinical symptoms } \\
\hline $\begin{array}{l}\text { Related clinical symptoms }, n(\%) \\
\text { No obvious symptoms, } n(\%)\end{array}$ & $\begin{array}{r}6(13.0) \\
40(87.0)\end{array}$ & $\begin{array}{c}0(0) \\
44(100)\end{array}$ \\
\hline \multicolumn{3}{|l|}{ Laboratory findings } \\
\hline White blood cell count, median (IQR), $10^{9} / \mathrm{L}$ & $6.3(5.2-7.1)$ & $5.7(4.5-7.5)$ \\
\hline Neutrophil ratio, median (IQR), \% & $73.0(68.1-78.3)$ & $67.7(61.6-77.1)$ \\
\hline Neutrophil count, median (IQR), $10^{9} / \mathrm{L}$ & $4.5(3.7-5.0)$ & $4.0(2.9-5.8)$ \\
\hline Lymphocyte ratio, mean (SD), \% & $14.9(5.5)$ & $18.8(8.0)$ \\
\hline Lymphocyte count, median (IQR), $10^{9} / \mathrm{L}$ & $0.9(0.6-1.2)$ & $1.1(0.8-1.2)$ \\
\hline Monocyte ratio, median (IQR), \% & $7.7(6.7-9.3)$ & $8.1(6.9-9.8)$ \\
\hline Monocyte count, median (IQR), $10^{9} / \mathrm{L}$ & $0.5(0.4-0.6)$ & $0.5(0.4-0.6)$ \\
\hline Hemoglobin, mean (SD), g/L & $112.7(20.4)$ & $106.4(16.9)$ \\
\hline Platelet, mean $(\mathrm{SD}), 10^{9} / \mathrm{L}$ & $172.5(52.6)$ & $182.4(65.2)$ \\
\hline \multicolumn{3}{|l|}{ Outcome } \\
\hline \multicolumn{3}{|l|}{ MHD patients } \\
\hline Critical patients, $n(\%)$ & $7(15.2)$ & 0 \\
\hline Cure, $n(\%)$ & - & $44(95.7)$ \\
\hline Death, $n(\%)$ & - & $2(4.3)$ \\
\hline Medical staff, $n$ & $n=63$ & $n=63$ \\
\hline Staff with COVID-19, $n(\%)$ & $6(9.5)$ & 0 \\
\hline Cure, $n(\%)$ & 0 & $6(9.5)$ \\
\hline Death, $n(\%)$ & 0 & 0 \\
\hline
\end{tabular}

Values are expressed as mean (standard deviation), median (25th-75th percentile) or $n$ (\%). COVID-19, coronavirus disease 2019; CT, computed tomography; IQR, interquartile range; SD, standard deviation; * Related clinical symptoms: fever, fatigue, cough, expectoration.

the upper, middle and lower lobes were 80.4, 52.2 and $76.1 \%$, respectively. Nineteen (41.3\%) MHD patients suspected of COVID-19 had ground-glass opacities on chest CT. In the SARS-CoV-2 nucleic-acid-positive and -negative groups, the proportion of unilateral pneumonia was 42.9 and $33.3 \%(p>0.05)$, and the proportion of bilateral pneumonia was 57.1 and $66.7 \%$ ( $p>0.05)$, respectively. As for the affected lobes, the proportion of all 5 lobes affected in the nucleic-acid-positive group was higher than that in the nucleic-acid-negative group (57.1 vs. $33.3 \%$ ), but there was no statistically significant difference $(p>0.05)$. The proportion of ground-glass opacity in chest CT of the two groups was 42.9 and $41 \%$, respectively $(p>0.05)$ (Table 2).

As for laboratory tests, the white blood cell count (5.4 vs. $6.5 \times 10^{9} / \mathrm{L}, p>0.05$ ), lymphocyte ratio (13.2 vs. $15.3 \%$, $p>0.05)$, and lymphocyte count $\left(0.5\right.$ vs. $0.7 \times 10^{9} / \mathrm{L}, p>$ $0.05)$ of the SARS-CoV-2 nucleic-acid-positive group were lower than those of the SARS-CoV-2 nucleic-acidnegative group. In general, there were no significant dif- ferences in age, sex, symptoms, CT findings, comorbidity and laboratory findings of SARS-CoV-2 nucleic-acidpositive and -negative patients (Table 2).

As mentioned above, 46 patients suspected of COVID-19 in our dialysis center were isolated in the dedicated area of the last shift of the dialysis unit, of whom 14 (30.4\%) COVID-19 (critically ill and confirmed) patients were transferred to COVID-19-designated hospitals. All patients considered as COVID-19 were followed up until April 1, 2020. During the epidemic period, 2 critically ill patients with COVID-19 died, with an overall mortality rate of $4.3 \%$. Except for the 2 patients who died, the other 44 patients had no clinical symptoms after isolation and treatment, and reexamination of chest CT showed no signs of viral pneumonia. After the treatment during the quarantine period, the lymphocyte count and ratio of the MHD patients were slightly higher than those before treatment, while the neutrophil count and ratio decreased. The results of other routine blood tests fluctuated slightly (Table 3 ). 
By February 15, 2020, 6 (9.5\%) of the 63 medical staff members studied were suspected of COVID-19 and 3 (4.8\%) medical staff were confirmed as COVID-19, and all of them were cured through treatment and were quarantined; no new infections occurred after quarantine was initiated (Table 3 ).

\section{Discussion}

In this retrospective study, we found that $10 \%$ of $\mathrm{MHD}$ patients and $6.0 \%$ of medical staff in the dialysis facilities of Wuhan were suspected of COVID-19, among which the proportion of MHD patients and medical staff confirmed by SARS-CoV-2 nucleic acid accounted for $1.7 \%$ and $2.9 \%$, respectively. The SARS-CoV-2 infection rates in MHD patients and related medical staff were relatively high in Wuhan. Due to the effects of various uremic toxins, impaired cellular and humoral immune functions, and the long-term protein energy malnutrition, patients with renal failure are poorly resistant to pathogens $[6,7]$. Therefore, HD patients are particularly vulnerable to respiratory pathogens and severe pneumonia. Kwan et al. [8] found that there was a higher morbidity of SARS in MHD patients than in the general population in 2003. In addition, HD patients have to commute from home to the dialysis center every other day, which places these patients at a higher risk of virus infection and might make SARS-CoV-2 infection more widespread. The infected proportions of the MHD patients (18.9\%) and medical staff (9.5\%) in our hospital were all much higher than the data from another hospital (1.2\%, total medical staff morbidity data of COVID-19 from Tongji Hospital). A lack of staff (doctors and nurses) might add to the problems in clinical work. The medical staff in dialysis facilities frequently closely contacted these MHD patients, which might place them at high risk of infection. On the other hand, the lack of personal protection equipment in the early stage of the epidemic made the situation worse.

Therefore, it is urgently necessary to take steps to diagnose and quarantine COVID-19 patients promptly. The sensitivity of the SARS-CoV-2 nucleic acid detection kits was much lower in the early stage of the epidemic in Wuhan, but chest CT screening for the patients was much more likely to be performed in China [9]. In this abominable situation, the New Coronavirus Pneumonia Prevention and Control Program (5th edition, in Chinese), published by the National Health Commission of China, recommended guidelines for the clinical diagnosis of $\mathrm{CO}$ VID-19 for individuals in the most affected area, Wuhan
[1]. All patients clinically diagnosed with COVID-19 were included in this study and strict quarantine measures were carried out regardless of whether these patients were positive for the SARS-CoV-2 nucleic acid test. These clinical diagnoses of COVID-19 patients were virtually the suspected COVID-19 patients according to the 7th edition guideline of China [2].

Chest CT manifestations of COVID-19 in MHD patients were similar to those in the general population, mainly including unilateral or bilateral pneumonia, multiple patchy ground-glass shadows, and other rare CT findings, including pulmonary consolidation $[10,11]$. Previous studies have shown that chest CT images changes rapidly in COVID-19 patients, and repeated CT examination is crucial for the identification of the disease and the monitoring of its development $[12,13]$. Fang et al. [14] found that the sensitivity of chest CT was higher than that of the SARS-CoV-2 nucleic acid test at initial patient presentation ( 98 vs. $71 \%, p<0.05$ ). For MHD patients, the clinical symptoms of COVID-19 were not typical, which might mean that most of them had mild cases; therefore, repeated chest CT examination was essential for the early identification and diagnosis of COVID-19. It was reported that some patients showed positive results only after a second, third or even more SARS-CoV-2 nucleic acid tests due to sampling or other reasons [14]. For all MHD patients clinically diagnosed with COVID-19 (suspected cases, according to the 7th edition of the guidelines), viral nucleic acid tests need to be repeated when conditions permit. Such aggressive diagnostic criteria and quarantine measures might lead to the misdiagnosis of some patients and increase the probability of false positives, but such aggressive quarantine measures could avoid the problems of missed diagnosis and the further spread of COVID-19 in dialysis centers. Of course, repeated detection of SARS-CoV-2 nucleic acid for those suspected patients to confirm COVID-19 would be performed during follow-up.

Among the primary diseases, the proportion of diabetes in the group of patients suspected of COVID-19 infection seemed much higher than that in the uninfected group [10 (21.7\%) vs. 22 (11.1\%), $p=0.054]$. Among the comorbidities, the proportion of patients with suspected COVID-19 infection with hypertension was significantly lower than that of patients without infection [28 (60.9\%) vs. 188 (94.9\%), $p<0.05$ ], which may indicate that MHD patients with diabetes were more likely to be infected with SARS-CoV-2 [15], and hypertension might play a positive role in preventing infection. This phenomenon requires further explanation. A possible explanation is that 
people with diabetes had metabolic disorders, and individuals with low body immunity were more likely to be infected with SARS-CoV-2 [16]. In addition, some MHD patients without high blood pressure might suffer from hypotension and malnourishment, which makes them prone to infection [17]. In the analysis of dialysis modes, the proportion of patients undergoing HDF in the uninfected group (22 [11.2\%]) seemed much higher than that in the suspected COVID-19 group (3 [6.5\%]), but the data were limited; therefore, we could not determine which mode of dialysis was better to prevent infection.

All MHD patients who tested positive for SARS-CoV-2 nucleic acid had mild or even no symptoms. Existing studies have shown that among patients with confirmed COVID-19 in the general population, approximately $77-$ $98 \%$ of patients had fever, $59-82 \%$ of patients had cough, and $40-70 \%$ of patients had fatigue and other related clinical symptoms [10, 18-20]. Compared with the general population, the clinical symptoms of MHD patients diagnosed with COVID-19 were not obvious and were atypi$\mathrm{cal}$, and most of them did not show hypoxemia. We found that $85.7 \%$ of the SARS-CoV-2-positive MHD patients and $87.0 \%$ of the MHD patients suspected of COVID-19 in our center had no obvious symptoms. It could be speculated that the immune dysfunction impaired by uremia did not react to viral invasion and/or that effective hemodialysis treatment might reduce the viral load in patients [21]. Our data showed that in the analysis of modes of dialysis, the group suspected of COVID-19 was different from the uninfected group $(p<0.05)$, but the data were limited. Previous studies have found that there were lower HBV and HCV loads after dialysis than before HD in HD patients $[22,23]$. The related mechanism was unclear, but it might be related to the destruction or clamping of virus particles on the dialyzer membrane during dialysis [23]. It is unclear whether HD itself could reduce the SARS-CoV-2 load, and the related mechanism needs further study. Moreover, plenty of asymptomatic cases demonstrated that some infected patients really were asymptomatic [24-26].

Regarding laboratory tests, there were significant differences between the suspected COVID-19 group and the uninfected group in neutrophil ratio, neutrophil count, lymphocyte ratio ( 14.9 vs. $20.1 \%, p<0.05$ ), lymphocyte count $\left(0.90 \times 10^{9} / \mathrm{L}\right.$ vs. $\left.1.0 \times 10^{9} / \mathrm{L}, p<0.05\right)$ and lactate dehydrogenase level. These data met our diagnostic criteria, especially those of the lymphocyte count and ratio. However, the lymphocyte count in all MHD patients decreased to $1.0 \times 10^{9} / \mathrm{L}$ (normal reference range 1.1-3.2 $\times$ $10^{9} / \mathrm{L}$ ), which might mean that all MHD patients had low immunity [27]. The level of the inflammatory factor hCRP in the two groups was higher than the normal reference range, but there was no difference between the two groups, which may be due to the long-term microinflammatory status in hemodialysis patients [28].

Undiagnosed COVID-19 in MHD patients might be a potential source of infection. The MHD units belong to the public area, with a high population and high fluidity. If MHD patients infected with COVID-19 are not detected and isolated in a timely manner, it might lead to an outbreak. All of the patients and medical staff in the HD facility were in close contact, which could put all at risk of infection. For example, in the Middle East respiratory syndrome (MERS) outbreak in Saudi Arabia from April 1 to May 23, 2013, a total of 21 out of 23 infections were acquired through human-to-human transmission in HD units, intensive care units, or inpatient units in three different health care facilities [29]. Wuhan was the area most seriously affected by the epidemic in China. Due to the shortage of medical resources in Wuhan in January, these patients suspected of COVID-19 were not isolated for timely treatment. However, these patients might have become a mobile source of infection, which is not conducive to the management and control of the disease. Since February 2020, after the aggressive quarantine of all patients suspected of COVID-19 in our dialysis center, no new medical staff members have been infected, and the infection rate of MHD patients has also decreased significantly. Through our lessons learned from the above experience, we hope to receive attention and support from the government and relevant departments in all areas with serious outbreaks to ensure that all MHD patients suspected of COVID-19 are quarantined and receive proper treatment. By doing so, we hope to reduce the infection rate of relevant medical staff and other exposed patients and control the spread of disease from the source.

There were some limitations to our experiment. First, there was a lack of data on demographics, clinical manifestations, and laboratory tests for patients in the $52 \mathrm{HD}$ centers in Wuhan. Second, our hospital lacked records of laboratory test results for lymphocytes and more frequent SARS-CoV-2 nucleic acid detection in MHD patients during the quarantine period. Third, due to the limitation of detection technique, SARS-CoV-2 antibody could not be detected in February.

In summary, this study indicated that the SARS-CoV-2 infection rates in MHD patients and related medical staff were relatively high in Wuhan. In MHD patients, the symptoms of COVID-19 might be less severe than those in the general population. To protect MHD patients and 
related medical staff, we recommended that all MHD patients suspected of COVID-19 could receive treatment and aggressive quarantine measures to reduce the infection rate of relevant medical staff and other exposed patients. More frequent detection of SARS-CoV-2 nucleic acid, antibody and chest CT should be performed in these high-risk groups.

\section{Acknowledgement}

The authors greatly appreciate all the hospital staff for their efforts in recruiting and treating patients and thank all patients involved in this study. Thanks to American Journal Experts for the modification of the manuscript.

\section{Statement of Ethics}

Written informed consent was obtained from patients before enrollment. The study protocol was approved by the Medical Ethics Committee of Tongji Hospital Affiliated to Tongji Medical College, Huazhong University of Science and Technology (No. TJC20200138).

\section{Disclosure Statement}

The authors have no conflicts of interest to declare.

\section{Funding Sources}

This work was financially supported by the National Natural Science Foundation of China (NSFC, Grants No. 81974089, 81470994, 81873556, 81470948, 81670633, 81570667), international (regional) cooperation and exchange projects (NSFC-DFG, Grant No. 81761138041), the Major Research plan of the National Natural Science Foundation of China (Grant No. 91742204), the National Key Research and Development Program (Grants 2016YFC0906103, 2018YFC1314003-1) and the National Key Technology R\&D Program (Grant 2015BAI12B07) and Science Foundation of Hubei Province (2018CFB554).

\section{Author Contributions}

J.L., F.H. and G.X. designed the study and drafted the manuscript. Y.Y. and M.G. were responsible for the data integrity and analysis. Y.Y. and F.X. provided statistical support. S.G. critically reviewed the manuscript. All authors provided significant inputs for data collection, intellectual content, and final approval.

\section{References}

1 China NHCo. The new coronavirus pneumonia prevention and control program. 5th ed. [cited 2020 Feb 4]. Available from: http://www.nhc.gov.cn/yzygj/s7653p/2020 02/3b09b894ac9b4204a79db5b8912d4440/ files/7260301a393845fc87fcf6dd52965ecb. pdf.

2 China NHCo. Guidelines for diagnosis and treatment of COVID-19. [cited 2020 Mar 3]. Available from: http://www.nhc.gov.cn/yzy$\mathrm{gj} / \mathrm{s} 7653 \mathrm{p} / 202003 / 46 \mathrm{c} 9294 \mathrm{a} 7 \mathrm{dfe} 4 \mathrm{cef} 80 \mathrm{dc7f5}$ 912 eb1989/files/ce3e6945832a438eaae 415350a8ce964.pdf.

3 Zhang L, Zuo L. Current burden of end-stage kidney disease and its future trend in China. Clin Nephrol. 2016 Supplement 1;86 (2016) (13):27-28.

4 Corman VM, Landt O, Kaiser M, Molenkamp R, Meijer A, Chu DK, et al. Detection of 2019 novel coronavirus (2019-nCoV) by real-time RT-PCR. Euro Surveill. 2020 Jan; 25(3).

5 WHO. Laboratory testing for 2019 novel coronavirus $(2019-\mathrm{nCoV})$ in suspected human cases. Interim guidance. Jan 17, 2020. Available from: https://www.who.int/publications-detail/laboratory-testing-for2019-novel-coronavirus-in-suspected-human-cases-20200117.

6 Descamps-Latscha B, Chatenoud L. T cells and $\mathrm{B}$ cells in chronic renal failure. Semin Nephrol. 1996 May;16(3):183-91.
7 Pesanti EL. Immunologic defects and vaccination in patients with chronic renal failure. Infect Dis Clin North Am. 2001 Sep;15(3): $813-32$.

8 Kwan BC, Leung CB, Szeto CC, Wong VW, Cheng YL, Yu AW, et al. Severe acute respiratory syndrome in dialysis patients. J Am Soc Nephrol. 2004 Jul;15(7):1883-8.

9 Ai T, Yang Z, Hou H, Zhan C, Chen C, Lv W, et al. Correlation of Chest CT and RT-PCR Testing in Coronavirus Disease 2019 (COVID-19) in China: A Report of 1014 Cases. Radiology. 2020 Feb:200642.

10 Chen N, Zhou M, Dong X, Qu J, Gong F, Han $\mathrm{Y}$, et al. Epidemiological and clinical characteristics of 99 cases of 2019 novel coronavirus pneumonia in Wuhan, China: a descriptive study. Lancet. 2020 Jan 30.

11 Chung M, Bernheim A, Mei X, Zhang N, Huang $M$, Zeng $X$, et al. CT Imaging Features of 2019 Novel Coronavirus (2019-nCoV). Radiology. 2020 Apr;295(1):202-7.

12 Bernheim A, Mei X, Huang M, Yang Y, Fayad ZA, Zhang N, et al. Chest CT Findings in Coronavirus Disease-19 (COVID-19): Relationship to Duration of Infection. Radiology. 2020 Feb:200463.

13 Pan F, Ye T, Sun P, Gui S, Liang B, Li L, et al. Time Course of Lung Changes On Chest CT During Recovery From 2019 Novel Coronavirus (COVID-19) Pneumonia. Radiology. 2020 Feb:200370
14 Fang Y, Zhang H, Xie J, Lin M, Ying L, Pang $\mathrm{P}$, et al. Sensitivity of Chest CT for COVID-19: comparison to RT-PCR. Radiology. 2020 Feb:200432

15 Guo W, Li M, Dong Y, Zhou H, Zhang Z, Tian $\mathrm{C}$, et al. Diabetes is a risk factor for the progression and prognosis of COVID-19. Diabetes Metab Res Rev. 2020 Mar:e3319.

16 Gupta S, Koirala J, Khardori R, Khardori N Infections in diabetes mellitus and hyperglycemia [vii.]. Infect Dis Clin North Am. 2007 Sep;21(3):617-38.

17 Katona P, Katona-Apte J. The interaction between nutrition and infection. Clin Infect Dis. 2008 May;46(10):1582-8.

18 Huang C, Wang Y, Li X, Ren L, Zhao J, Hu $\mathrm{Y}$, et al. Clinical features of patients infected with 2019 novel coronavirus in Wuhan, China. Lancet. 2020 Feb 15;395(10223): 497-506.

19 Wang D, Hu B, Hu C, Zhu F, Liu X, Zhang J, et al. Clinical Characteristics of 138 Hospitalized Patients With 2019 Novel CoronavirusInfected Pneumonia in Wuhan, China. JAMA. 2020 Feb 7.

20 Xu XW, Wu XX, Jiang XG, Xu KJ, Ying LJ, Ma $\mathrm{CL}$, et al. Clinical findings in a group of patients infected with the 2019 novel coronavirus (SARS-Cov-2) outside of Wuhan, China: retrospective case series. BMJ. 2020 Feb; 368:m606. 
21 Tullis RH, Duffin RP, Zech M, Ambrus JL Jr. Affinity hemodialysis for antiviral therapy. I. Removal of HIV-1 from cell culture supernatants, plasma, and blood. Ther Apher. 2002 Jun;6(3):213-20.

22 Noiri E, Nakao A, Oya A, Fujita T, Kimura S. Hepatitis $\mathrm{C}$ virus in blood and dialysate in hemodialysis. Am J Kidney Dis. 2001 Jan;37(1): $38-42$.

23 Fabrizi F, Lunghi G, Alongi G, Aucella F, Barbisoni F, Bisegna S, et al. Kinetics of hepatitis $B$ virus load and haemodialysis: a prospective study. J Viral Hepat. 2008 Dec;15(12):91721.
24 Meng H, Xiong R, He R, Lin W, Hao B, Zhang $\mathrm{L}$, et al. CT imaging and clinical course of asymptomatic cases with COVID-19 pneumonia at admission in Wuhan, China. J Infect. 2020 Apr;S0163-4453(20)30211-5.

25 Pan X, Chen D, Xia Y, Wu X, Li T, Ou X, et al. Asymptomatic cases in a family cluster with SARS-CoV-2 infection. Lancet Infect Dis. 2020 Apr;20(4):410-1.

26 Setti L, Kirienko M, Dalto SC, Bonacina M, Bombardieri E. FDG-PET/CT findings highly suspicious for COVID-19 in an Italian case series of asymptomatic patients. Eur J Nucl Med Mol Imaging. 2020 Apr.
27 Saad K, Elsayh KI, Zahran AM, Sobhy KM. Lymphocyte populations and apoptosis of peripheral blood $\mathrm{B}$ and $\mathrm{T}$ lymphocytes in children with end stage renal disease. Ren Fail. 2014 May;36(4):502-7.

28 Ren H, Zhou X, Luan Z, Luo X, Han S, Cai Q, et al. The Relationship between Carotid Atherosclerosis, Inflammatory Cytokines, and Oxidative Stress in Middle-Aged and Elderly Hemodialysis Patients. Int J Nephrol. 2013;2013:835465.

29 Assiri A, McGeer A, Perl TM, Price CS, Al Rabeeah AA, Cummings DA, et al.; KSA MERSCoV Investigation Team. Hospital outbreak of Middle East respiratory syndrome coronavirus. N Engl J Med. 2013 Aug;369(5):407-16. 